Meta

Journal des tradlucteurs

Translators' Journal

\title{
Termes et concepts de la classologie
}

\section{Édouard Natanson}

Volume 21, numéro 2, juin 1976

URI : https://id.erudit.org/iderudit/003696ar

DOI : https://doi.org/10.7202/003696ar

Aller au sommaire du numéro

Éditeur(s)

Les Presses de l'Université de Montréal

\section{ISSN}

0026-0452 (imprimé)

1492-1421 (numérique)

Découvrir la revue

Citer cet article

Natanson, É. (1976). Termes et concepts de la classologie. Meta, 21(2), 134-140. https://doi.org/10.7202/003696ar d'utilisation que vous pouvez consulter en ligne.

https://apropos.erudit.org/fr/usagers/politique-dutilisation/ 


\section{Termes et concepts de la classologie}

\section{Objet de l'analyse}

C'est une bonne règle terminologique que d'attribuer à chaque concept son terme propre. En effet, il est aussi incommode qu'imprécis d'assigner une seule dénomination à deux concepts appartenant au même domaine concret. En application de cette règle, nous allons mettre en corrélation les concepts et les termes de la classologie ${ }^{1}$.

Notre étude cherche d'abord à découvrir les lacunes et les imprécisions des langages scientifiques et techniques actuels. Des éléments de solution théorique sont ensuite proposés en vue d'une éventuelle normalisation de la terminologie du classement. Enfin, nous tentons d'expliquer les principes permettant d'opérer une sélection rationnelle parmi les termes soumis à l'examen.

\section{Les concepts}

Soit une collection de livres qu'il s'agit de ranger dans un ordre quelconque. N'importe laquelle de leurs propriétés peut servir à organiser l'ensemble ; il suffit de considérer chaque livre (chaque élément de l'ensemble) en fonction des critères (les propriétés) retenus.

Imaginons maintenant une autre situation. Un livre arrive dans une bibliothèque, et on le range à la place qui lui revient dans une classification préétablie. Cette opération est différente de la précédente. En effet, la première vise à imposer un ordre à un ensemble d'objets, et la seconde, à rattacher un élément à un ensemble défini par avance. Dans un cas, on divise un ensemble, dans l'autre, on intègre un élément dans l'ensemble.

Cette distinction conceptuelle établie, considérons maintenant deux autres aspects du classement d'un système de classement, et le rangement des éléments en fonction de ce système particulier - et non pas d'un autre. La distance qui sépare la première opération de la seconde est aussi grande que celle qui sépare l'établissement d'un devis de la construction d'un bâtiment d'après ce devis. Est-il nécessaire de rappeler que ces opérations sont toujours confiées à des spécialistes différents ?

1. Classologie. Discipline nouvelle comprenant la théorie, les méthodes et les procédés du classement ainsi que la création de systèmes de classement. 
Il est enfin souhaitable de considérer l'opposition entre les concepts suivants : 《action d'établir un système de classement » et « résultat de cette action ». Il ne s'agit pas simplement de rappeler une vérité bien connue, à savoir que l'action ne doit pas être confondue avec son résultat, mais de préciser que les deux concepts en cause appartiennent à des catégories conceptuelles différentes : action et objet, car, en effet, le concept de système, qui nous intéresse, se rattache à la catégorie des objets ${ }^{2}$.

En résumé, les concepts dont nous aurons à tenir compte au cours de l'analyse qui va suivre sont les suivants :

1. Action de diviser un ensemble en vue d'effectuer un classement;

2. Action de définir la place d'un élément, d'après ses propriétés spécifiques, dans un système préétabli ;

3. Action d'établir un système de classement ;

4. Le système de classement résultant de cette action. Nous devrons en outre prendre en considération :

5. La personne qui range les objets d'après le système ;

6. L'appareil à l'aide duquel on range les objets d'après le système ;

7. La personne qui établit les systèmes de classement ;

8. La discipline qui englobe la théorie, les méthodes et les procédés de classement ;

9. Le spécialiste de la théorie, des méthodes et des procédés de classement ;

10. Le contenant (meuble, boîte ou portefeuille) servant à garder les objets classés.

Chacun de ces concepts sera l'occasion de considérations terminologiques.

\section{La terminologie}

Les termes en usage pour désigner les concepts définis ci-dessus comprennent les verbes classer et classifier avec leurs dérivés classement, classification, classeur, classificateur, etc. Mais l'usage courant, qui tolère de nombreux cas de polysémie est cependant trop vague pour convenir à une terminologie scientifique, qui exige, on le sait, des définitions d'une précision rigoureuse. Il suffit, pour s'en convaincre, d'examiner les dictionnaires les plus connus. Voici ce qu'on y trouve :

«CLASSER - 1. Diviser en classes, en catégories ; dérivés : classement, classeur.

2. Mettre dans un certain ordre ${ }^{3}$.

«CLASSEMENT - Action de ranger dans un certain ordre, résultat de cette action ${ }^{4} \$$.

2. Les catégories conceptuelles sont : objet, action, état, propriété. Chaque catégorie comporte des sous-catégories.

3. Paul Robert, Dictionnaire alphabétique et analogique de la langue française, t. I, Paris, 1969, p. 793 .

4. Ibid. 
«CLASSEUR - Portefeuille ou meuble à compartiments qui sert à classer des papiers ${ }^{5} \gg$.

- CLASSIFIER - Faire, établir les classifications ; dérivé : CLASSIFICATEUR - celui qui établit des classifications ${ }^{6}$. $\gg$

«CLASSIFICATION - Action de distribuer par classes, par catégories; résultat de cette action ${ }^{7}$.

Et dans un autre dictionnaire :

«CLASSER - Ranger par classes, par catégories... mettre dans un certain ordre $^{8}$. 》

«CLASSEMENT - Action de classer ${ }^{9} . »$

« CLASSEUR - Personne qui classe des objets, des documents, etc. Objet ou meuble de bureau divisé en compartiments, servant à classer les papiers, les dessins, etc. ${ }^{10}$ »

"CLASSIFIER - Ranger par classes, par catégories ${ }^{11}$.»

"CLASSIFICATION - Action de distribuer par classes, par catégories ${ }^{12}$. "

«CLASSIFICATEUR, - TRICE - Qui établit des classifications, qui sait répartir par classes, catégories, etc. ${ }^{13}{ }$

Ce tableau est fort décevant. Les deux définitions du verbe classer sont contradictoires, car «diviser en classes 》 ne désigne pas la même opération que « ranger par classes». En effet, si l'on peut «diviser en classes» un ensemble, on ne peut « ranger par classes » que des éléments.

D'autre part, le Grand Larousse encyclopédique définit dans les mêmes termes les verbes classer et classifier, ce qui pourrait laisser croire qu'on se trouve en présence de deux parfaits synonymes, alors qu'en réalité chacun d'eux recouvre une réalité différente.

Quant au Dictionnaire alphabétique et analogique de la langue française, il désigne à l'aide d'un seul et même terme, classification, le fait d'établir un système de classement et le résultat de cette action, confondant ainsi deux concepts appartenant à des catégories différentes, celle de l'action et celle de l'objet. Le Grand Larousse ne mentionne même pas le deuxième sens de classification (le résultat). Or, c'est précisément le terme de classification qui convient le mieux au concept de "système de classement», puisque des expressions telles que classification périodique, classification décimale, classification zoologique, etc. ont d'ores et déjà introduit ce sens dans l'usage.

5. Dictionnaire alphabétique et analogique de la langue française, t. I, p. 793.

6. Ibid., p. 794.

7. Ibid., p. 793.

8. Grand Larousse encyclopédique, t. 3, Paris, 1970, p. 175.

9. Ibid., p. 174.

10. lbid., p. 175 .

11. Ibid.

12. Ibid.

13. Ibid. 
Classer et classifier étant des synonymes, dans le Grand Larousse, on ne s'étonnera pas de constater que leurs dérivés, classement et classification correspondent à un seul concept, défini tantôt comme « action de classer », tantôt comme "action de distribuer par classes », ce qui revient au même puisqu'il s'agit toujours d'une opération portant sur les éléments de l'ensemble.

Les deux derniers termes de la collection, classeur et clasificateur ont des significations différentes lorsqu'ils sont employés pour désigner des personnes, classeur s'appliquant à celle qui range les objets, et classificateur, à celle qui établit un système de classement.

Classeur, enfin, selon le Robert, ne désigne que des objets (meubles ou portefeuilles), tandis que, d'après le Grand Larousse, il peut aussi dénoter des personnes. Ajoutons pour mémoire que, toujours d'après le Grand Larousse, un classeur dans l'industrie papetière est un appareil «destiné au classage de la cellulose ou de la pâte ${ }^{14} \gg$. À côté de cela, on trouve une classeuse totalisatrice, "machine de bureau permettant de classer et d'endosser les chèques, effets ou pièces comptables, et d'en lister et totaliser les montants sur les bandes individuelles correspondant aux cases de classement, d'une part, et sur une bande de contrôle, d'autre part ${ }^{15}$ ». Ainsi, dans les grands dictionnaires contemporains, le terme de classeur est attribué à trois concepts d'ordre différent : le spécialiste, le meuble et l'appareil. Dans tous les cas, il s'emploie au féminin comme au masculin.

Signalons que dans une des définitions étudiées, on trouve classage employé à la place de classement pour signifier l'action de classer.

Sans doute ce tissu de contradictions et d'inconséquences n'est-il pas dû aux lexicographes, dont le rôle se borne à enregistrer l'usage et à consigner dans les dictionnaires le résultat de leurs observations. À l'issue de cette brève analyse, on constate trois lacunes: l'action de définir la place d'un élément, d'après ses propriétés spécifiques, dans un système préétabli (paragr. $2,2^{\circ}$ ), la discipline qui englobe la théorie, les méthodes et les procédés de classement (ibid., $8^{\circ}$ ), et le spécialiste de la théorie, des méthodes et des procédés de classement (ibid., $9^{\circ}$ ) n'ont pas de nom dans le lexique.

\section{Néologismes}

De telles lacunes devaient forcément susciter la création de néologismes. Dans la revue Industries et techniques ${ }^{16}$, on relève classologue repris dans la Clé des mots ${ }^{17}$, pour désigner le spécialiste du classement. Si le spécialiste est un classologue, il nous semble que l'étude à laquelle il se livre pourrait bien être la classologie, suivant le modèle qui fait correspondre zoologie et zoologue, biologie et biologiste, géologie et géologue, etc. Voilà donc le lexique du classement enrichi de deux termes.

14. Grand Larousse encyclopédique, t. 3, p. 175.

15. Ibid.

16. Industries et techniques, 235 (octobre 1973) : 123.

17. La Clé des mots, C.I.L.F., cahier du juin 1974, fiche $n^{\circ} 1463$. 
Moins heureuse nous paraît cependant l'introduction de classette, petite valise métallique destinée à recevoir des dossiers suspendus. Ce diminutif, s'il peut convenir à l'objet en question, s'applique mal, d'autre part, aux meubles de proportions plus considérables dans lesquels sont habituellement rangés les objets classés.

\section{Le concept de système et son rôle dans la classologie}

Sans doute serait-il souhaitable que tous les termes du lexique de la terminologie du classement fussent formés à l'aide du radical CLASS -, mais à défaut d'une famille de mots de type idéal, on peut, pour éviter de former des néologismes par trop artificiels, recourir à des mots comportant un autre radical, pourvu que soient respectés certains critères logiques. On aura soin, par exemple, de n'emprunter que des termes appartenant à un domaine plus vaste que celui du classement proprement dit, car l'inverse pourrait entraîner des résultats fâcheux. Cette constatation est valable dans tous les domaines. Si tout spécialiste de l'hydraulique peut être qualifié de mécanicien, le contraire n'est pas vrai, car tous les mécaniciens ne sont pas des hydrauliciens, l'hydraulique n'étant qu'une branche d'une science plus vaste, la mécanique.

La discipline qui englobe la classologie est la systématologie. Pour savoir s'il est possible d'utiliser le radical SYSTEM - examinons les termes qui en dérivent et leurs concepts. Ce radical provient du grec, sustêma signifiant « ensemble, composition $\gg$ et connaît de nombreux dérivés. Quant au concept qu'il recouvre, nous n'en citerons que les définitions les plus générales, et partant les plus abstraites, données par les deux dictionnaires qui ont servi de source à la présente étude :

a) «Réunion de principes coordonnés de façon à former un tout scientifique ou un corps de doctrine ${ }^{18}$.

b) «Ensemble organisé d'éléments intellectuels ${ }^{19}$. 》

La première de ces définitions est, à notre avis, trop limitative, car le système de Linné, par exemple, n'est pas une réunion de principes, les principes (nous dirions les critères) ont seulement servi à la compréhension des relations entre les éléments et au classement de ces mêmes éléments.

La deuxième définition appelle aussi quelques précisions supplémentaires. En effet, pourquoi n'y est-il question que d' « éléments intellectuels $»$ ? Notre milieu écologique n'est-il pas un système ? Et une machine ? Et un appareil de téléphone? Ce sont tous des systèmes concrets; les éléments des systèmes et les systèmes eux-mêmes peuvent être de nature idéelle aussi bien que matérielle. Lorsqu'un ingénieur établit le plan d'une machine, cette machine en tant que système n'existe encore que sous la forme d'un ensemble d'idées, mais une fois réalisée, elle prend la forme d'un ensemble d'éléments concrets.

18. Grand Larousse encyclopédique, t. 10, Paris, 1970, p. 123.

19. Paul Robert, Dictionnaire alphabétique et analogique de la langue française, t. 6, Paris, 1969, p. 444. 
Ceci posé, il ne nous reste plus qu'à proposer une définition du concept de système: «ensemble organisé d'éléments se trouvant en interrelation, en interdépendance, ou en interaction ».

Il découle de cette définition qu'un ensemble de classes, après étude, devient un système qu'on appelle classification. La classification est donc une espèce de système intellectuel. C'est d'ailleurs pourquoi la «science des classifications des formes vivantes ${ }^{20} \gg$ s'appelle la systématique.

Par extension de sens, systématicien désigne non seulement le spécialiste de la systématique, mais aussi le spécialiste des classifications en général ${ }^{21}$. À défaut d'un mot comportant le radical CLASS - , ce terme pourrait bien désigner la personne qui établit les systèmes de classement.

Une autre observation confirme encore l'étroite liaison du radical SYSTEM avec les concepts de la classologie. Dès 1843, en effet, l'appellation de systématisation est appliquée à la notion de "réunion en un système ${ }^{22}$ » ou, autrement dit, « action de systématiser ${ }^{23}{ }$. Mais puisque l'établissement d'un système est compris dans l'action de systématiser, systématisation peut fort bien tenir lieu d'un mot de la famille de CLASS - pour signifier l'action d'établir un système de classes.

\section{Le concept de TRIEUR (-EUSE)}

Les progrès de la technique ont permis de mécaniser le classement d'objets divers, par exemple, celui des envois postaux d'après leur destination. Les appareils qui effectuent ce classement devraient être désignés par un terme comportant la racine CLASS-, mais selon les dictionnaires, le terme classeuse-totalisatrice est déjà affecté à une espèce particulière d'appareils. Son hyperonyme est trieur (ou trieuse), dont voici quelques définitions :

a) «TRIEUSE - Machine à trier [...]. Machine mécanographique capable de classer rapidement des cartes perforées ${ }^{24}$.

b) \& TRIEUR, EUSE - [...] Appareil ou dispositif de bureau permettant de trier des documents en un certain nombre de catégories ou selon leurs destinataires ${ }^{25}$.

D'après son étymologie, ce terme ne correspond pas exactement au concept qu'il désigne. En effet, Bloch et Wartburg ont fait remarquer qu'en français le verbe trier (du lat. triare) désigne l'action de séparer: \&Le sens de TRIER s'explique par le fait qu'on broie le grain pour en séparer les parties inutilisables ${ }^{26}$. » Or, la séparation est compromise dans le classement qui suppose encore la répar-

20. Dictionnaire alphabétique et analogique de la langue française, t. 6, p. 444.

21. Ibid.

22. Ibid., p. 445.

23. Grand Larousse encyclopédique, t. 10, p. 122.

24. Dictionnaire alphabétique et analogique de la langue française, t. 6, p. 661 .

25. Grand Larousse encyclopédique, t. 10, p. 497.

26. O. Bloch, W. von Wartburg, Dictionnaire étymologique de la langue française, $5^{\circ}$ Edition, Paris, Presses Universitaires de France, 1968, p. 650. 
tition. Les termes de TRIAGE et de CLASSEMENT ne peuvent donc pas, à notre avis, être considérés comme équivalents. Mais, en raison de l'usage établi, trieur (ou trieuse) qui désigne l'appareil servant au classement du charbon, du minerai, du grain, d'envois postaux, etc., bien qu'hétérogène aux points de vue formel et sémantique, par rapport au système envisagé, peut être retenu. Cette solution nous semble plus heureuse que celle qui consiste à utiliser un seul terme polysémique comme, par exemple, calculatrice (personne et appareil), chargeur (personne et dispositif), etc.

\section{Terminologie systématique}

En conclusion, selon la règle «un concept, un terme », nous nous permettons de proposer la délimitation conceptuelle suivante des termes existants ainsi que de quelques néologismes :

\section{CONCEPTS}

Action de diviser un ensemble d'éléments en vue d'un classement.

Personne qui accomplit cette action.

Appareil à l'aide duquel on range les objets d'après le système de classement.

Action d'établir un système de classement.

Personne qui établit les systèmes de classement.

Action de définir la place d'un élément dans un ensemble d'après un système préétabli.

Discipline qui embrasse la théorie, les méthodes et les procédés du classement.

Spécialiste de la théorie, des méthodes et des procédés du classement. Meuble, boite ou portefeuille qui sert à garder des objets classés.

TERMES

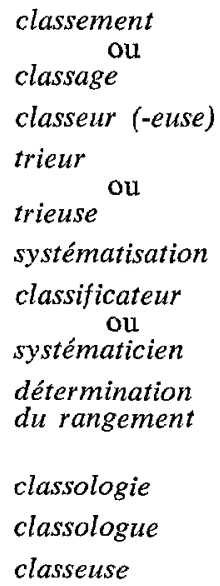

Le dernier terme proposé nécessite quelques explications, car nous l'avons déjà cité comme l'une des deux appellations pouvant être attribuées au concept de personne. Les deux formes, CLASSEUR et CLASSEUSE correspondent aux spécialistes, homme et femme ; l'emploi de ces formes est donc naturel et ne présente aucun problème du point de vue de la délimitation conceptuelle. S'il s'agit, cependant d'un meuble, d'une boîte ou d'un portefeuille, classeuse ne nous semble pas convenir, parce que les substantifs en -EUSE désignent en général des agents, c'est-à-dire des personnes et des appareils qui effectuent des opérations, ce que ne font pas les meubles, les boîtes et les portefeuilles. Cependant, compte tenu du fait que les termes CHAUFFEUSE, LISEUSE et COIFFEUSE désignent déjà des meubles, nous croyons possible d'admettre le terme de CLASSEUSE, bien qu'il soit alors polysémique.

De toute façon les attributions de termes proposées ci-dessus pourraient, à notre avis, servir à une délimitation plus nette des concepts que celles qui existent déjà dans l'usage actuel.

Édouard Natanson 